\title{
Quasigeostrophy against the wall
}

\author{
A. Venaille \\ Univ Lyon, Ens de Lyon, Univ Claude Bernard, CNRS, Laboratoire de Physique, F-69342 \\ Lyon, France
}

(Received xx; revised xx; accepted xx)

Coasts are obstructions to the classical derivation of continuously stratified quasigeostrophic equations, due to possible resonances between slow internal coastally trapped Kelvin waves and anticyclones. [Deremble et al Ocean Modelling 2017] proposed a coupled model between a quasigeostrophic interior and boundary layer Kelvin wave dynamics. We revisit the derivation of this model, paying particular attention to conservation laws. We find that quasigeostrophic energy is conserved despite the existence of Kelvin wave shocks in the boundary layer. The effect of those shocks is to change the global distribution of potential vorticity, and, consequently the interior flow structure. In that respect, we show that there is an active control of the boundary region on the interior flow.

Key words: Quasigeostrophic flows, Kelvin waves, hydraulic jumps, cyclone-anticyclone asymmetry

\section{Introduction}

Quasigeostrophic models play a prominent role in our understanding of midlatitude atmospheric and oceanic dynamics (Vallis 2017). They describe the slow evolution of geostrophically balanced motion, filtering out the fast dynamics of inertia-gravity waves. Yet, in a series of recent papers, Dewar and collaborators showed that geostrophically balanced motion in continuously stratified fluid may interact with slow internal Kelvin waves trapped along a lateral wall, and this is an obstruction to the classical derivation of quasigeostrophic equations: Dewar \& Hogg (2010) identified a mechanism of potential vorticity injection in interior flows through the formation Kelvin wave shocks; Dewar et al. (2011) addressed the relevance of this process within the oceanic energy cycle; Hogg et al. (2011) deciphered how and when Kelvin wave shocks are generated by an initially geostrophic flow, following previous work on rotating hydraulics (Pratt \& Whitehead 2007). Building upon these results, Deremble et al. (2017) proposed a coupled model between interior quasigeostrophic dynamics and a boundary layer equation describing nonlinear Kelvin wave dynamics. The so-called Deremble-JohnsonDewar model captured the generation of cyclones by shocks following the impact of an anticyclone on a coast. This mechanism of potential vorticity generation by shocks bears similarities with rip-current formation in the surf zone (Peregrine 1998; Bühler 2000), albeit at a different scale. The main difference here is that Kelvin wave shocks only produce cyclones. Deremble et al. (2017) emphasised the key role of this boundary layer dynamics in shaping the interior flow properties close to the wall. They also found via numerical simulations that this process acts as a significant sink of energy, but without providing scaling with respect to the Rossby number, the small parameter of the asymptotic model.

The aim of this paper is to clarify how global conservation laws of standard, unbounded 
quasigeostrophic models are affected by the presence of a coast, by revisiting the derivation of Deremble et al. (2017). The paper is organised as follows. We introduce in a second section the hydrostatic, rotating Boussinesq equations, and we explain why the presence of a wall makes the derivation of quasigeostrophic equations difficult. Starting from the multiple layer shallow water model with sufficiently thin layer thickness, a new derivation of Deremble-Johnson-Dewar model is proposed in a third section, paying particular attention to mass conservation, energy conservation, and a local model for potential vorticity injection by shocks. We end in a fourth section with a discussion on symmetries and on possible geophysical applications.

\section{Boussinesq syllabus}

\subsection{Hydrostatic Boussinesq dynamics on the f-plane}

Our starting point is the 3D Boussinesq, hydrostatic equations with traditional approximation for the Coriolis force (Vallis 2017). This is a standard model for geophysical flows, including the effect of rotation and stratification through the Coriolis parameter $f$ (twice the projection of the planet rotation rate on the local vertical axis) and the buoyancy frequency $N$. Calling $L$ and $H$ the typical horizontal and vertical length scales of the flow with typical velocity $U$, the Boussinesq dynamics admits three non-dimensional parameters: the aspect ratio, the Rossby number and the Burger number, defined as

$$
\alpha \equiv \frac{H}{L}, \quad \text { Ro } \equiv \frac{U}{L f}, \quad \mathrm{Bu} \equiv\left(\frac{N H}{f L}\right)^{2} .
$$

The hydrostatic limit corresponds to $\alpha \ll 1$. The hydrostatic Boussinesq system is

$$
\begin{aligned}
\partial_{x} u+\partial_{y} v+\partial_{z} w & =0 \\
0 & =-\partial_{z} p+b \\
\operatorname{Ro}\left(\partial_{t}+u \partial_{x}+v \partial_{y}+w \partial_{z}\right) u & =-\partial_{x} p+v \\
\operatorname{Ro}\left(\partial_{t}+u \partial_{x}+v \partial_{y}+w \partial_{z}\right) v & =-\partial_{y} p-u \\
\operatorname{Ro}\left(\partial_{t}+u \partial_{x}+v \partial_{y}+w \partial_{z}\right) b & =-\operatorname{Bu} w
\end{aligned}
$$

The field $b$ is the perturbation buoyancy corresponding to rescaled density anomalies around the stable stratification. To simplify the discussion, we consider the case where $f$ and $N$ are constant.

\subsection{Plane waves}

We first consider a case without boundary, and look for solutions of the hydrostatic Boussinesq equations linearized around a state of rest. Eigenmodes are on the form $e^{i \omega t-i k_{x} x-i k_{y} y-i k_{z} z}$, and the problem admits three wave bands with dispersion relations

$$
\omega= \pm \frac{1}{\mathrm{Ro}} \sqrt{1+\frac{\mathrm{Bu}}{k_{z}^{2}}\left(k_{x}^{2}+k_{y}^{2}\right)}, \quad \omega=0 .
$$

For a given $k_{z}$, we recover the dispersion relation of shallow water waves with celerity $c=N /\left|k_{z}\right|$, see figure 1]. The zero frequency wave band corresponds to geostrophic modes, for which the pressure force balance the Coriolis force. The non-zero frequency bands corresponds to hydrostatic, internal inertia-gravity waves. Geostrophic modes and inertia-gravity wave modes are separated by a frequency gap of width $\mathrm{Ro}^{-1}$. The existence of this gap is central to the classical derivation of the quasigeostrophic model. 

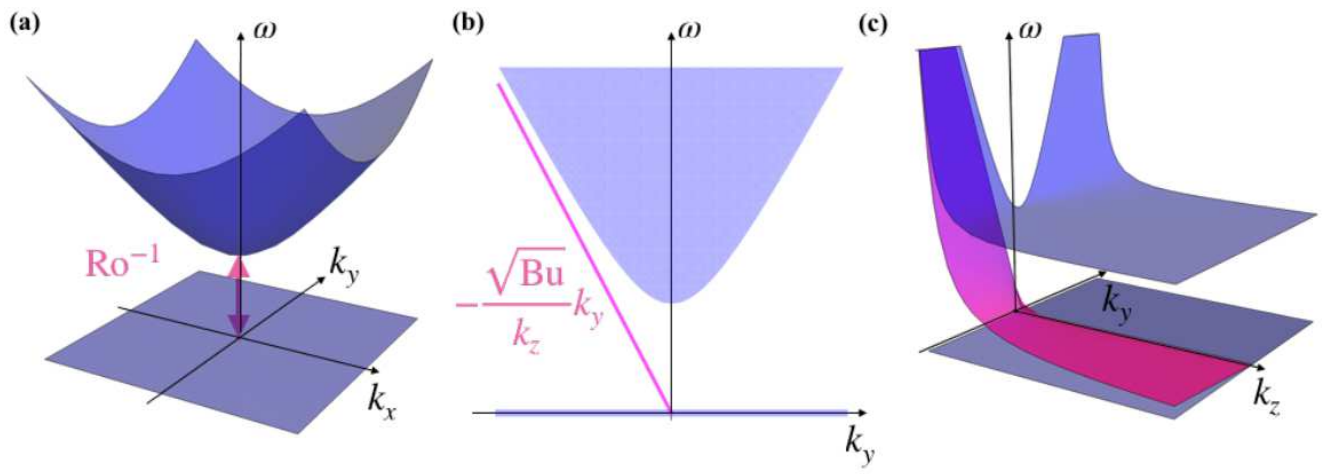

Figure 1. Dispersion relation of hydrostatic Boussinesq model linearized around a state of rest, adapted from (Zeitlin 2018). (a) Unbounded case, $k_{z}$ fixed. There is a frequency gap $\Delta \omega=f$ between the inertia-gravity wave band and the (flat) geostrophic wave band. (b) Coastal case (wall at $x=0$ ), $k_{z}$ fixed. The dispersion relation for varying values of $k_{x}$ is projected in $k_{y}, \omega$ plane. The magenta line corresponds to coastally trapped internal Kelvin waves. (c) Coastal case, $k_{z}>0$. The blue surface at the top represents the bottom boundary of the inertia-gravity wave band. The magenta surface corresponds to Kelvin-wave dispersion relation. When $k_{z}$ tends to $\infty$ for a given value of $k_{y}$, the Kelvin wave dispersion relation tends to the flat geostrophic band. This is an obstruction to classical derivation of quasigeostrophic equations.

\subsection{Unbounded quasigeostrophic dynamics}

The dynamics of geostrophic modes can be decoupled from the dynamics of internal gravity waves modes in the small Rossby number limit Ro $\ll 1$. This amounts to consider a wide frequency gap limit between (slow) geostrophic and (fast) internal inertia-gravity wave modes. The quasigeostrophic model describes the slow dynamics of geostrophic mode. It is derived through an asymptotic expansion, with a small parameter given by the Rossby number $R o \ll 1$, for a fixed Burger number $B u \sim 1$ (Vallis 2017). This last condition means that typical horizontal flow structures $L$ are of the order of an intrinsic length scale named, the Rossby radius of deformation $N H / f$.

\subsection{Internal coastal Kelvin waves}

The presence of a lateral wall allows for the along-wall propagation of a new class of waves trapped in the across-wall direction, with frequencies filling the frequency gap between inertia-gravity waves and geostrophic modes. Those are the celebrated internal coastal Kelvin waves. Their salient features are derived from the hydrostatic Boussinesq model linearized around a state of rest, in the presence of a lateral (vertical) wall along the $y$-direction. We take the wall at $x=0$ and consider the flow taking place in the region $x>0$, with impermeability boundary condition at the wall: $u(0, y, z, t)=0$. Eigenmodes are of the form $g(x) e^{i \omega t-i k_{y} y-i k_{z} z}$ with $g(x)$ to be determined. There is two classes of eigenmodes. First, the bulk modes, with $g(x)=\sin \left(k_{x} x\right)$ and with the same dispersion relation as in the unbounded case (2.7). Second, an additional branch of boundary modes that correspond to internal coastal Kelvin waves, satisfying geostrophic balance in the along-wall direction with vanishing velocity in the across-wall direction:

$$
v=\partial_{x} p, \quad \text { with } \partial_{x x} p=-\mathrm{Bu}^{-1} \partial_{z z} p, \quad u=0 .
$$

Those modes have several features that will play an central role in the derivation of a model coupling interior and boundary dynamics: they are trapped along the wall, 
unidirectional, and propagate as non-rotating hydrostatic internal gravity waves:

$$
g(x)=e^{-x / l}, \quad l=\frac{\mathrm{Bu}^{1 / 2}}{\left|k_{z}\right|}, \quad \omega=-\frac{\mathrm{Bu}^{1 / 2}}{\mathrm{Ro}} \frac{k_{y}}{\left|k_{z}\right|} .
$$

Both the trapping length scale and the phase speed vanish for large vertical wavenumbers.

We readily see on the dispersion relation plotted figure 1 that the presence of a new branch of Kelvin wave modes filling the frequency gap is an obstruction to the classical derivation of quasigeostrophic dynamics: whatever the value of the horizontal wave number $k_{x}$ and the value of the frequency $\omega$, there is a value of vertical wavenumber $k_{z}$ such that a coastal wave exists. This means that one can not dismiss the presence of coastal waves when performing the standard multiple scale expansions leading to quasigeostrophic dynamics.

Let us consider a Kelvin waves with wavenumber $k_{y} \sim 1$ and frequency $\omega$. Interactions between this wave and geostrophic modes having a typical eddy turnovertime $L / U \sim 1$ occur when $\omega \sim 1$. Injecting this scaling in Eq. (2.9) and assuming $\mathrm{Bu} \sim 1$ leads to $1 / k_{z} \sim$ Ro and $l \sim$ Ro (with dimensions, this gives $1 / k_{z}^{*} \sim \operatorname{Ro} H$ and $l^{*} \sim \operatorname{Ro} L$ ). To conclude, the linear analysis offers important physical insights on possible coupling between the bulk (interior) geostrophic modes and the boundary (coastally trapped) Kelvin waves in the limit of vanishing Rossby numbers, with three properties that will be essential features of Deremble-Johnson-Dewar model:

- The interactions involves internal Kelvin waves having a vertical wavelength that scales linearly with the Rossby number, and being confined in a boundary layer with a thickness that also scales linearly with the Rossby numbers.

- For all the coastal Kelvin waves, the across wall velocity $u$ is identically zero. This property will hold at lowest order in the amplitude of the wave for a superposition of coastally trapped modes interacting nonlinearly, since the nonlinear term in the evolution of $u$ is proportional to $u$.

- Since there is only one coastally trapped mode for a given value of $\left(k_{y}, k_{z}\right)$, the boundary layer dynamics that describes the nonlinear evolution of these coastally trapped waves will be governed by a $2 \mathrm{D}$ equations in the $(y, z)$ plane.

\section{Coupling a quasigeostrophic interior to Kelvin wave dynamics}

We now revisit the derivation of the Deremble-Johnson-Dewar model that couples an interior quasigeostrophic flow to boundary layer Kelvin wave dynamics in the limit $\mathrm{Ro} \rightarrow 0$ with $\mathrm{Bu} \sim 1$. While their derivation was performed in the continuously stratified case with isopycnal coordinates, our starting point is the multiple layer shallow water. This is the natural discretisation of isopycnal hydrostatic Boussinesq equations, keeping track of the layerwise potential vorticity conservation. From a practical point of view, this makes direct connections with numerical simulations that deal with discretised models. From a fundamental or pedagogical perspective, this makes possible a direct application of previous results on rotating shallow water hydraulics (Pratt \& Whitehead 2007; Zeitlin 2018). The continuous case in density coordinate is recovered in the limit of vanishing layer thickness.

The multiple-layer shallow water model is written as a triplet of dynamical equations for each layer $i$ (with $i$ increasing upward) with depth independent horizontal velocity 
$\mathbf{u}_{i}=\left(u_{i}, v_{i}\right)$ and thickness $h\left(1+\delta \eta_{i}\right)$ :

$$
\begin{aligned}
\operatorname{Ro}\left(\partial_{t}+\mathbf{u}_{i} \cdot \nabla\right) u_{i} & =-\partial_{x} p_{i}+v_{i} \\
\operatorname{Ro}\left(\partial_{t}+\mathbf{u}_{i} \cdot \nabla\right) v_{i} & =-\partial_{y} p_{i}-u_{i} \\
\left(\partial_{t}+\mathbf{u}_{i} \cdot \nabla\right) \delta \eta_{i} & =-\left(1+\delta \eta_{i}\right) \nabla \cdot \mathbf{u}_{i}
\end{aligned}
$$

which express momentum conservation and mass conservation, respectively. Interface thickness variations and pressure fields are related through hydrostatic balance (see Appendix A):

$$
\delta \eta_{i}=-\frac{\mathrm{Ro}}{\mathrm{Bu}} \frac{p_{i+1}-2 p_{i}+p_{i-1}}{(\delta z)^{2}}, \quad \delta z=\frac{h}{H},
$$

with a constant density jump $\Delta \rho / \rho_{0}$ between adjacent layers, such that $g \Delta \rho /\left(\rho_{0} h\right)=N^{2}$. Realistic configurations would also require specific equations for the upper and lower layers (interpreted as upper and lower boundary conditions in the continuous limit); we focus here on internal layers to simplify the discussion, assuming that the domain is unbounded in the vertical (the layers are then indexed by $i \in \mathbb{Z}$ ). The mean interface thickness must be chosen sufficiently thin to allow for possible resonances between interior geostrophic modes and boundary Kelvin waves identified by the linear analysis performed in section 2: $h=O(\mathrm{Ro})$. Note that for a finite depth ocean, this would require $N \sim 1 / \mathrm{Ro}$ layers.

As in section 2, the flow domain takes place in a semi-infinite horizontal domain, with fields vanishing at infinity, and an impermeability constraint at the wall:

$$
u_{i}(0, y, t)=0 \text {. }
$$

We assume that the initial flow satisfies quasigeostrophic scaling, with horizontal scale, vertical scale and velocities of order one, interface height variations of order Ro, corresponding to vertical pressure variation between adjacent layers scaling as $\delta z$. The strategy is to divide the domain into an interior region satisfying standard quasigeostrophic equations, and a boundary layer with typical thickness scaling as Ro.

\subsection{Quasigeostrophic dynamics in the interior}

The interior dynamics is derived from Eqs. (3.1]3.2 3.3.3.4 following standard procedure based on asymptotic expansion in a low Ro limit (Vallis 2017), with the ansatz

$$
\left[\mathbf{u}_{i}, p_{i}\right]=\left[\mathbf{u}_{i, 0}^{g}, p_{i, 0}^{g}\right]+\operatorname{Ro}\left[\mathbf{u}_{i, 1}^{g}, p_{i, 1}^{g}\right]+O\left(\mathrm{Ro}^{2}\right)
$$

We also assume that typical vertical variations of the pressure fields (up to order one) between adjacent layers is of order $\delta z$, consistently with the assumption of an initial condition satisfying quasigeostrophic scaling. According to Eq. (3.4), interior interface height variations scale linearly with Ro.

- At order 0, one gets geostrophic balance, and hydrostatic balance still holds:

$$
\mathbf{u}_{i, 0}^{g}=\left(-\partial_{y} \psi_{i}, \partial_{x} \psi_{i}\right), \quad \psi_{i} \equiv p_{i, 0}^{g}, \quad \eta_{i, 0}^{g}=-\frac{1}{\mathrm{Bu}} \frac{p_{i+1,0}^{g}-2 p_{i, 0}^{g}+p_{i-1,0}^{g}}{\delta z^{2}}
$$

- At order 1 we recover quasigeostrophic dynamics

$$
\partial_{t} q_{i}^{g}+\mathbf{u}_{i, 0}^{g} \cdot \nabla q_{i}^{g}=0, \quad q_{i}^{g} \equiv \nabla^{2} \psi_{i}+\mathrm{Bu}^{-1}(\delta z)^{-2}\left(\psi_{i+1}-2 \psi_{i}+\psi_{i}\right) .
$$

At this stage, one can not integrate the dynamics in Eq. (3.8) for two reasons, both of them related with potential vorticity inversion: (i) the boundary condition for $\psi$ at the wall remains unknown; (ii) one can not rule out a source of vorticity within the boundary 
layer that would affect the streamfunction outside the boundary layer. To address those two issues, it is necessary to dwell into Kelvin boundary layer dynamics.

\subsection{Kelvin wave dynamics in the boundary layer}

According to the analysis of linearized hydrostatic Boussinesq dynamics in section 2, the Kelvin boundary layer dynamics is expected to be confined in a region of size Ro $L$ away from the wall, with vertical variations of the fields taking place over a distance RoH. This motivates the following change of variable:

$$
X=\frac{x}{\mathrm{Ro}}, \quad \delta Z=\frac{\delta z}{\mathrm{Ro}} .
$$

The velocity and pressure fields in the boundary layer are decomposed as follows:

$$
\begin{aligned}
& v_{i}=v_{i}^{b}(X, y, t)+\left.v_{i, 0}^{g}\right|_{x=0}(y, t)+\left.\operatorname{Ro} v_{i, 1}^{g}\right|_{x=0}(y, t)+\left.\operatorname{Ro} X \partial_{x} v_{i, 0}^{g}\right|_{x=0}(y, t)+O\left(\operatorname{Ro}^{2}\right), \\
& u_{i}=u_{i}^{b}(X, y, t)+\left.u_{i, 0}^{g}\right|_{x=0}(y, t)+\left.\operatorname{Ro} u_{i, 1}^{g}\right|_{x=0}(y, t)+\left.\operatorname{Ro} X \partial_{x} u_{i, 0}^{g}\right|_{x=0}(y, t)+O\left(\operatorname{Ro}^{2}\right), \\
& p_{i}=p_{i}^{b}(X, y, t)+\left.p_{i, 0}^{g}\right|_{x=0}(y, t)+\left.\operatorname{Rop}_{i, 1}^{g}\right|_{x=0}(y, t)+\left.\operatorname{Ro} X \partial_{x} p_{i, 0}^{g}\right|_{x=0}(y, t)+O\left(\operatorname{Ro}^{2}\right) .
\end{aligned}
$$

The matching condition between inner (index "b" for boundary) and outer (interior quasigeostrophic) solution is

$$
\lim _{X \rightarrow+\infty}\left[u_{i}^{b}, v_{i}^{b}, p_{i}^{b}\right]=[0,0,0] .
$$

The boundary fields are also expanded as

$$
\left[\mathbf{u}_{i}^{b}, p_{i}^{b}\right]=\left[\mathbf{u}_{i, 0}^{b}, p_{i, 0}^{b}\right]+\operatorname{Ro}\left[\mathbf{u}_{i, 1}^{b}, p_{i, 1}^{b}\right]+O\left(\mathrm{Ro}^{2}\right),
$$

and it will be convenient to decompose the total velocity and pressure fields as

$$
\left[\mathbf{u}_{i}, p_{i}\right]=\left[\mathbf{u}_{i, 0}, p_{i, 0}\right]+\operatorname{Ro}\left[\mathbf{u}_{i, 1}, p_{i, 1}\right]+O\left(\mathrm{Ro}^{2}\right) .
$$

The fields $\left[\mathbf{u}_{i, 0}, p_{i, 0}\right]$ and $\left[\mathbf{u}_{i, 1}, p_{i, 1}\right]$ include the trace of the interior field in the boundary layer regions as defined in Eq. (3.10, 3.11 3.12).

Special care must be taken to evaluate the different terms in the expansion of interface height variations $\delta \eta_{i}$ defined in Eq. (3.4). Indeed, we have assumed that vertical variations of interior pressure between adjacent layers scale as $\delta z$, and, based on linear analysis, we anticipated that vertical variations of boundary pressure between adjacent layers scale as $\delta Z$. Thus, the interface height variations can be expressed as

$$
\delta \eta_{i}=\delta \eta_{i, 0}+O(\mathrm{Ro}), \quad \delta \eta_{i, 0}=-\frac{1}{\mathrm{Bu}} \frac{p_{i+1,1}^{b}-2 p_{i, 1}^{b}+p_{i-1,1}^{b}}{(\delta Z)^{2}} .
$$

Now that we have introduced the ansatz for the solution in the boundary layer, we write down the rescaled dynamical equations. Momentum equations read

$$
\begin{aligned}
& \operatorname{Ro}\left(\partial_{t}+\operatorname{Ro}^{-1} u_{i} \partial_{X}+v_{i} \partial_{y}\right) u_{i}=-\operatorname{Ro}^{-1} \partial_{X} p_{i}+v_{i}, \\
& \operatorname{Ro}\left(\partial_{t}+\operatorname{Ro}^{-1} u_{i} \partial_{X}+v_{i} \partial_{y}\right) v_{i}=\quad-\partial_{y} p_{i}-u_{i} .
\end{aligned}
$$

It will be convenient to use potential vorticity as a third dynamical equation:

$$
\left(\partial_{t}+\operatorname{Ro}^{-1} u_{i} \partial_{X}+v_{i} \partial_{y}\right) q_{i}=0, \quad q_{i}=\frac{1+\zeta_{i}}{1+\delta \eta_{i}}, \quad \zeta_{i} \equiv \partial_{X} v_{i}-\operatorname{Ro}_{y} u_{i} .
$$

Consistently with the assumption of an initial condition satisfying quasigeostrophic scaling, material conservation of potential vorticity for a fluid particle with initial relative 
vorticity $\zeta_{i}^{(t=0)} \sim$ Ro and initial interface thickness variation $\delta \eta_{i}^{(t=0)} \sim$ Ro can be recast as

$$
\zeta_{i}-\delta \eta_{i}=O(\mathrm{Ro})
$$

We inject the ansatz (3.10,3.1133.12 3.6 3.14 in the rescaled dynamical system 3.17 3.18 3.20) and collect terms at each order with respect to Ro.

- At order -1, the momentum equation in $X$-direction yields

$$
\partial_{X} p_{i, 0}=0
$$

- At order 0 , the momentum and potential vorticity equations yield respectively

$$
\begin{aligned}
u_{i, 0} \partial_{X} u_{i, 0} & =-\partial_{X} p_{i, 1}+v_{i, 0}, \\
u_{i, 0} \partial_{X} v_{i, 0} & =-\partial_{y} p_{i, 0}-u_{i, 0}, \\
\partial_{X} v_{i, 0}-\delta \eta_{i, 0} & =0 .
\end{aligned}
$$

Differentiating Eq. (3.23) by $X$ and using Eq. (3.21) leads to $\partial_{X}\left(u_{i, 0}\left(\partial_{X} v_{i, 0}+1\right)\right)=0$. Using the impermeability condition (3.5) leads then to $u_{i, 0}\left(\partial_{X} v_{i, 0}+1\right)=0$ for all $X$. The case $\partial_{X} v_{i}=-1$ corresponds to a vanishing interface thickness, i.e. $1+\delta \eta_{i, 0}=0$, according to Eq. (3.24). This may occur along shock lines. From now on, we describe the flow dynamics away from these isolated singularities. This corresponds to the second case $u_{i, 0}(X, y, t)=0$. Using the matching condition (3.13), we find an impermeability condition for the geostrophic (interior) velocity field, and a vanishing across-wall velocity in the boundary layer:

$$
\left.u_{i, 0}^{g}\right|_{x=0}=0, \quad u_{i, 0}^{b}(X, y, t)=0
$$

Eq. (3.23) is now further simplified as $\partial_{y} p_{i, 0}=0$. Using this equation together with Eq. (3.21) and the matching condition (3.13) yields

$$
p_{i, 0}^{b}=0,\left.\quad p_{i, 0}^{g}\right|_{x=0}(y, t)=\psi_{i, \text { wall }}(t) .
$$

The second equality is the standard impermeability condition for quasigeostrophic flows along a wall. The value of $\psi_{i \text {,wall }}$ will be determined using layerwise global mass conservation later on. Finally, Eq. (3.22) and (3.24) boil down to

$$
v_{i, 0}^{b}=\partial_{X} p_{i, 1}^{b}, \quad \frac{\partial^{2}}{\partial X^{2}} p_{i, 1}^{b}=-\frac{1}{\mathrm{Bu}} \frac{p_{i+1,1}^{b}-2 p_{i, 1}^{b}+p_{i-1,1}^{b}}{(\delta Z)^{2}} .
$$

This shows that the triplet of boundary layer fields $\left[\mathbf{u}_{i, 0}^{b}, p_{i, 0}^{b}\right]$ satisfies the polarization relation of coastal Kelvin waves, as in Eq. (2.8). The boundary fields are then fully prescribed by the amplitude of $v_{i}^{b}$ at the boundary $X=0$. Their dynamics is obtained at next order.

- At order 1 , the momentum equation in the $y$-direction evaluated at the wall yields

$$
\text { At } X=0: \partial_{t} v_{i, 0}+\partial_{y}\left(\frac{1}{2} v_{i, 0}^{2}+p_{i, 1}\right)=0
$$

We have used $u_{i}(0, y, t)=0$ and the order-1 impermeability constraint. Eq. (3.28) can be recast as a dynamical evolution for $v_{i, 0}^{b}(0, y, t)=v_{i, 0}(0, y, t)-v_{i, 0}^{g}(0, y, t)$, assuming that geostrophic fields are known. Noticing that $\left.\partial_{y} p_{i, 1}\right|_{X=0}=\left.\partial_{y} p_{i, 1}^{b}\right|_{X=0}(y, t)$, the combination of Eq. (3.27) with Eq. (3.28) and boundary condition (3.13) provide the system of equations derived in Deremble et al. (2017).

\subsection{Potential vorticity production by shallow water shocks}

Dewar \& Hogg (2010); Hogg et al. (2011); Deremble et al. (2017) showed that the 
boundary layer dynamics lead to shocks and the concomittant creation of cyclonic vorticity. Based on global conservation of circulation, Deremble et al. (2017) proposed a model for the feedback of these shocks on the interior quasigeostrophic dynamics. We propose here a more local justification of their model, relying on the theory of rotating shallow water shocks (Peregrine 1998; Pratt \& Whitehead 2007; Zeitlin 2018).

A shallow water shock line, in the $X$-direction indexed by $s$ in layer $i$ and located at $y=y_{s, i}(t)$ is associated with a jump of the Bernoulli potential across the shock, see e.g. (Zeitlin 2018):

$$
\left[B_{i}\right] \equiv B_{i}\left(X, y_{s, i}^{+}\right)-B_{i}\left(X, y_{s, i}^{-}\right), \quad B_{i}\left(X, y_{s, i}^{ \pm}\right) \equiv \frac{\mathbf{u}_{i}^{2}\left(X, y_{s, i}^{ \pm}\right)}{2}+p_{i}\left(X, y_{s, i}^{ \pm}\right)
$$

When the value of $\left[B_{i}\right]$ varies along the shock, in the $X$ direction,there is a jump of potential vorticity across the shock (Zeitlin 2018):

$$
\left[q_{i}\right] \equiv q_{i}\left(X, y_{s, i}^{+}\right)-q_{i}\left(X, y_{s, i}^{-}\right)=\operatorname{Ro}^{-1} \frac{\partial_{X}\left(\left[B_{i}\right]-\dot{y}_{s, i}\left[v_{i}\right]\right)}{h_{i}\left(v_{i}-\dot{y}_{s, i}\right)},
$$

where $\left[v_{i}\right]=v_{i}\left(X, y_{s, i}^{+}, t\right)-v_{i}\left(X, y_{s, i}^{-}, t\right)$ is the velocity jump across the shock, $\dot{y}_{s, i} \equiv$ $\mathrm{d} y_{s, i} / \mathrm{d} t$ is the shock velocity and $h_{i}\left(v_{i}-\dot{y}_{s, i}\right)$ is the mass flux through the shock for an observer moving with the shock. This mass flux is conserved across the shock, with $\left[h_{i}\left(v_{i}-\dot{y}_{s, i}\right)\right]=0$. The combination of a potential vorticity jump and a constant mass flux through the shock implies a net production of potential vorticity per unit time and per unit shock length, see e.g. (Zeitlin 2018). The total amount of potential vorticity production at $y=y_{s, i}$ in the boundary layer region is thus

$$
\int_{0}^{+\infty} \mathrm{d} X \partial_{X}\left(\left[B_{i}\right]-\dot{y}_{s, i}\left[v_{i}\right]\right)=\dot{y}_{s, i}\left[v_{i}\right]_{X=0}-\left[B_{i}\right]_{X=0},
$$

where the r.h.s. is the jump evaluated at $\left(X=0, y=y_{s, i}\right)$. We have used the fact that there is no shock in the (quasigeostrophic) interior, for $X \rightarrow+\infty$.

The net production of potential vorticity in the boundary layer contradicts our assumption of materially conserved potential vorticity used to derive the Kelvin wave dynamics in the boundary layer. One way to have a self-consistent model taking into account the local inviscid production of vorticity at $y=y_{s, i}$ is to inject in Eq. (3.8) the total amount of potential vorticity of Eq. (3.31), at a distance $x=\sqrt{\mathrm{Ro}}$ much larger than the boundary layer of size Ro, while remaining asymptotically close to the wall:

$$
\partial_{t} q_{i}^{g}+\mathbf{u}_{i}^{g} \cdot \nabla q_{i}^{g}=\delta(x-\sqrt{\mathrm{Ro}}) \delta\left(y-y_{s, i}\right)\left(\dot{y}_{s, i}\left[v_{i}\right]_{X=0}-\left[B_{i}\right]_{X=0}\right) .
$$

This infinitesimal shift of potential vorticity production from the boundary layer to the interior region is the only phenomenological step of the model derivation. It is motivated by numerical simulations showing production of cyclones through the detachment of boundary layers close to the shock location in primitive equation models (Deremble et al. 2017). This can also be interpreted as the continuous version of the discrete numerical algorithm used by (Deremble et al. 2017) to simulate their coupled reduced model: for a given grid size, the location of the source term at $x=\sqrt{\mathrm{Ro}}$ guarantees that potential vorticity injection occurs within the cell adjacent to the wall in the limit $\mathrm{Ro} \rightarrow 0$.

To be consistent with this procedure of potential vorticity injection in the interior following the formation of shocks in the boundary, the total circulation in the boundary regions must be left invariant, which, assuming that it is initially zero, implies

$$
\Gamma_{i}=\Gamma_{i}^{g}, \quad \text { with } \Gamma_{i} \equiv-\int_{-\infty}^{+\infty} \mathrm{d} y v_{i, 0}, \quad \Gamma_{i}^{g} \equiv-\int_{-\infty}^{+\infty} \mathrm{d} y v_{i, 0}^{g} .
$$




\subsection{Mass conservation, quasigeostrophic circulation, and final set of equations}

The full dynamical system coupling boundary dynamics with quasigeostrophic interior

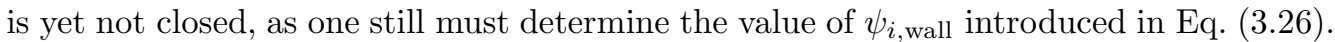
This is settled by using layerwise, global mass conservation:

$$
\left\langle\delta \eta_{i}\right\rangle=0, \quad \text { with }\left\langle\delta \eta_{i}\right\rangle \equiv \int_{0}^{+\infty} \mathrm{d} x \int_{-\infty}^{+\infty} \mathrm{d} y \delta \eta_{i} .
$$

The difficulty with respect to classical quasigeostrophic models is that variations of mass in the boundary layers are of the same order as variation of mass in the interior. Despite this subtlety, the use of Eq. (3.33) allows us to recover the constraint (see Appendix B):

$$
\left\langle\psi_{i+1}+\psi_{i-1}-2 \psi_{i}\right\rangle=0 .
$$

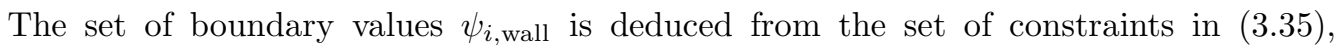
following standard procedure (McWilliams 1977). Let us note that Eq. (3.35) implies instantaneous adjustment of the mass in each interior layer. The reasons is that we assumed previously that quasigeostrophic motion has typical vertical scale of order 1 (size $H$ in dimensional units), and that Kelvin waves associated with vertical variations of order 1 are filtered out in the asymptotic expansion. Note that our phenomenological procedure of potential vorticity injection is such that structures of vertical size much smaller than $H$ can be formed in the interior, depending on shock properties in the boundary layer. This injection procedure induces therefore an inconsistency with respect to the initial hypothesis on the size of interior flows. By applying mass conservation (3.35), we continue to assume instantaneous adjustment for those smaller scale structures in the interior. Possible resonances between slow Kelvin wave dynamics and interior quasigeostrophic flow is still taken into account through the boundary layer equation (3.28).

Finally, the full coupled system is given by potential vorticity advection in Eq. (3.32) and Kelvin wave dynamics in Eq. (3.28). The interior velocity field is obtained by inversion of the quasigeostrophic potential vorticity field defined in Eq. (3.8), using the lateral boundary conditions in Eq. (3.26) and the constraints (3.35). Kelvin wave dynamics in Eq. (3.28) depends on the geostrophic interior field evaluated at the boundary; in turn, Eq. (3.28) is used to find shock locations and evaluate the corresponding velocity and Bernoulli potential jumps appearing in the r.h.s. of Eq. (3.32), and defined in Eq. (3.29). Thus, the knowledge of potential vorticity production is bound to the knowledge of Bernoulli potential jumps across shocks in the boundary layer dynamics. We have up to now not explained how to determine the actual value of such Bernoulli potential jumps. In the case of a one-layer shallow water model, one just needs to apply standard local mass and momentum conservation across the shock. However, the problem is indeterminate

in the case of multiple-layer shallow water flows, and no universal rule exist (Zeitlin 2018). One then either needs to introduce additional phenomenological assumptions on the shock behavior, or to bypass this issue by regularising the boundary Kelvin wave dynamics with dissipative terms. In the latter case, the Bernoulli potential jumps is estimated numerically across quasi-shocks that are defined at locations where gradients exceed a given threshold; this is the approach followed in (Deremble et al. 2017), who introduced viscous dissipation in Eq. (3.28).

\subsection{Conservation of quasigeostrophic energy}

Let us now consider the case of a finite-depth ocean with $N$ layers of thickness $h$, such that $N h=1$, with a rigid lid approximation (at layer $i=N$ ) and flat bottom boundary condition (at layer $i=1$ ). The dynamics is the same as in the case of an infinite number 
of layers, with two additional constraints related to upper and lower boundary conditions, namely $\psi_{N+1}=\psi_{N}$ and $\psi_{0}=\psi_{1}$, respectively. The quasigeostrophic energy is defined as

$$
E^{g} \equiv \frac{h}{2} \sum_{i=1}^{N}\left\langle\left(\nabla \psi_{i}\right)^{2}+\frac{1}{\mathrm{Bu}}\left(\frac{\psi_{i}-\psi_{i-1}}{\delta z}\right)^{2}\right\rangle .
$$

We assume the presence of $n_{i}$ shocks in each layer $i$. The shocks are indexed by $(s, i)$ with $1 \leqslant s \leqslant n_{i}$. Their location in the $y$ direction is denoted $y_{s, i}(t)$, and the corresponding potential vorticity injection rate is $\gamma_{s, i} \equiv \dot{y}_{s, i}\left[v_{i}\right]-\left[B_{i}\right]$, see Eq. (3.32). The temporal evolution of quasigeostrophic energy is computed by using the dynamical equation (3.32), the definition of quasigeostrophic potential vorticity in Eq. (3.8), as well as the definition of circulation in Eq. (3.33) and mass conservation in Eq. (3.35):

$$
\frac{\mathrm{d}}{\mathrm{d} t} E^{g}=h \sum_{i=1}^{N}\left(-\psi_{i, \text { wall }} \frac{\mathrm{d} \Gamma_{i}^{g}}{\mathrm{~d} t}+\sum_{s=1}^{n_{i}} \gamma_{s, i} \psi_{i}\left(\sqrt{\mathrm{Ro}}, y_{s, i}, t\right)\right), \quad \frac{\mathrm{d}}{\mathrm{d} t} \Gamma_{i}^{g}=\sum_{s=1}^{n_{i}} \gamma_{s, i} .
$$

Following the conventions used for the asymptotic analysis, the initial energy and the initial circulations are of order one; according to this asymptotic analysis, the potential vorticity injection rate $\gamma_{s, i}$ is also of order one. Using $h N \sim 1$ and $\psi_{i}\left(\sqrt{\mathrm{Ro}}, y_{s, i}\right)=$ $\psi_{i \text {, wall }}+\left.\sqrt{R o} \partial_{x} \psi\right|_{0, y_{s, i}}+o(\sqrt{\mathrm{Ro}})$, we find that the total quasigeostrophic energy vanishes in the limit Ro $\rightarrow 0$. We conclude that quasigeostrophic energy is conserved, unless the asymptotic approach fails in such a way that $\gamma_{s, i}$ scales as $1 / \sqrt{\mathrm{Ro}}$. This result does not contradict the observation of enhanced dissipation in the presence of a coast (Deremble et al. 2017). It just suggests that the amplitude of enhanced dissipation should tend to zero with Ro, so that the corresponding energy sink in the actual ocean would be a finite-Ro effect. It will be interesting to investigate how enhanced dissipation actually scales with the Ro in numerical models.

\section{Discussion and conclusion}

We have revisited the derivation of Deremble-Johnson-Dewar model coupling interior continuously stratified quasigeostrophic fluid to a boundary layer with low-frequency Kelvin wave dynamics. The boundary layer thickness scales linearly with the Rossby number, and the dynamics inside this layer is described by a two-dimensional dynamical equation at the wall. This wall dynamics leads to shocks. Our contribution is to clarify the matching condition between interior and boundary dynamics through mass conservation and shock properties, and to show that quasigeostrophic energy is conserved: shocks are an inviscid sink of energy, but those sinks are confined in a narrow boundary layer whose width scales linearly with Ro, so that their net contribution vanishes in the small Ro limit.

The original set of hydrostatic Boussinesq equations on the $f$-plane breaks time reversal symmetry. The symmetry breaking parameter is the Rossby number Ro. The quasigeostrophic model on the unbounded $f$-plane is derived in the limit $\operatorname{Ro} \rightarrow 0$. the Rossby number is not a parameter of this reduced model. Time-reversal symmetry is thus recovered in $f$-plane quasigeostrophic equations. The addition of a wall allows for the propagation of unidirectional Kelvin waves that bring back broken time-reversal symmetry into quasigeostrophic dynamics. This broken symmetry manifests itself in the interior flow as the formation of quasigeostrophic cyclones along the coast by Kelvin wave shocks: just as surface boundary layers favour cyclonic structures (Roullet \& Klein 2010), lateral Kelvin boundary layers break cyclone-anticyclone symmetry. 
Cyclones injected at the boundary start to impact the anticyclonic interior flow when the vertically integrated interior anticyclonic circulations become of the same order as the total amount of injected potential vorticity. Since injection takes place over a vertical scale of order Ro with a circulation production rate of order one, the interaction time can be estimated as $T_{\text {int }} \sim \mathrm{Ro}^{-1}$. The validity of the model in this long-time limit remains to be proven: shocks inject in the interior cyclonic structures with vertical size of order Ro, which seems to contradict the initial assumption of quasigeostrophic structures with order one vertical variations (scale $H$ in dimensional units). At a phenomenological level, one could argue that inverse cascade and barotropization processes organize the initially shallow cyclones into deeper ones.

This paper focused on inviscid dynamics and thus left aside the role of viscous boundary layers. In two-dimensional turbulence, the detachment of these layers may lead to dissipative structures (Nguven Van Yen et al. 2018), and drastically changes the interior vorticity dynamics (Roullet \& McWilliams 2014). The role of viscous boundary layers in continuously stratified rotating flows remains to be addressed.

While quasigeostrophic energy remains a conserved quantity at lowest order in Ro, boundary layer Kelvin dynamics plays an active role on the interior flow patterns, through the injection of cyclonic vorticity close to the coast. This could be a key aspect of oceanic western boundary currents detachment (Deremble et al. 2017). The $f$-plane coastal problem can also be interpreted as a toy model for the dynamics of equatorial planetary flows with symmetric temperature fields. Deremble-Johnson-Dewar mechanism could offer in this framework an explanation for the generation of intense equatorial cyclonic dipolar structures. Such patterns are an essential feature of Madden-Julian oscillations (Rostami \& Zeitlin 2019). For this reason, we think that Deremble-JohnsonDewar mechanism for the production of sub-mesoscale oceanic structures also deserves attention in the context of equatorial atmospheric flows.

\section{Acknowledgement}

This work has been motivated by a presentation in the working group GDT MathsInFluids organized by C. Lacave, D. Bresh and L. Saint-Raymond. A.V. warmly thank Bruno Deremble for fruitful discussions, Pierre Delplace for help with Mathematica, and the three reviewers who helped to improve significantly the presentation. Funding: ANR18-CE30-0002-01 WTF, and MSOM project.

\section{Declaration of interests}

The author reports no conflict of interest.

\section{Appendix A: hydrostatic relations for multiple-layer models}

We consider an ocean model with $N$ fluid layers. The layers are indexed in the upward direction by $i$, with $1 \leqslant i \leqslant N$. We assume a constant atmospheric pressure $P_{a}$ above the upper layer $i=N$. The total pressure fields, the thickness fields and the density fields in each layer are denoted $P_{i}(x, y, z, t), h_{i}(x, y, t), \rho_{i}=\rho_{0}+(N-i) \Delta \rho$, respectively. The interface elevation relative to a rest state and the interface depth between layers $i$ and $i+1$ are denoted $\eta_{i+0.5}$ and $z_{i+0.5}$. In dimensional units, interface height elevations, interface depth, and interface thickness are related through

$$
z_{i+0.5}=(i-N) h+\eta_{i+0.5} \quad h_{i}=h\left(1+\delta \eta_{i}\right), \quad \delta \eta_{i} \equiv \frac{\eta_{i+0.5}-\eta_{i-0.5}}{h} .
$$


The pressure is deduced from hydrostatic balance:

$$
P_{i}-P_{a}=g \rho_{i}\left(z_{i+0.5}-z\right)+\sum_{j=i+1}^{N} g \rho_{j} h_{j},
$$

For $1<i<N$, a straightforward computation yields to

$$
P_{i+1}-2 P_{i}-P_{i-1}=-g h \Delta \rho\left(\delta \eta_{i}+1\right) .
$$

We now introduce the rescaled dynamical pressure $p_{i} \equiv P_{i} /\left(\rho_{0} U f L\right)+F_{i}(z)$. This field is defined in each layer up to a function of $z$, since only its horizontal gradient matters in the dynamics. We choose the gauge function $F_{i}(z)$ in such a way to cancel the constant term in the r.h.s. of Eq. (4.3). Recalling that $g \Delta \rho h /\left(\rho_{0} f U L\right)=(h / H)^{2} \mathrm{Bu} / \mathrm{Ro}$, we obtain Eq. (3.4). This relation hold whatever the scaling of $N$ and $h$ with Ro.

\section{Appendix B: Global mass conservation}

Global conservation of mass in each layer $i$ is expressed in Eq. (3.34). We decompose the integral in the $x$ direction as a boundary term and an interior term:

$$
\int_{0}^{+\infty} \mathrm{d} x \delta \eta=\int_{0}^{\mathrm{Ro}^{3 / 4}} \mathrm{~d} x \delta \eta+\int_{\mathrm{Ro}^{3 / 4}}^{+\infty} \mathrm{d} x \delta \eta .
$$

Changing variable in the first integral with $X=x /$ Ro and considering the small Ro limit yields, at order Ro:

$$
\int_{0}^{+\infty} \mathrm{d} X \int_{-\infty}^{+\infty} \mathrm{d} y \delta \eta_{i, 0}+\int_{0}^{+\infty} \mathrm{d} x \int_{-\infty}^{+\infty} \mathrm{d} y \delta \eta_{i, 1}^{g}=0
$$

where $\delta \eta_{i, 0}$ is the order zero interface height variation in the boundary region and $\delta \eta_{i, 1}^{g}$ is the order one interior geostrophic interface height variation defined in Eq. (3.7). Variations of mass in the boundary layers are of the same order as in the interior since interface height variation $\delta \eta$ scales as Ro over a a region of size 1 in the quasigeostrophic region, while $\delta \eta$ scales as 1 over a region of size Ro in the boundary layer. Using the expression of $\eta_{i, 0}^{g}$ in Eq. (3.7), injecting Eq. (3.24) in Eq. (4.2), mass conservation reads

$$
\int_{0}^{+\infty} \mathrm{d} x \int_{-\infty}^{+\infty} \mathrm{d} y\left(\psi_{i+1}+\psi_{i-1}-2 \psi_{i}\right)=\operatorname{Bu} \delta z^{2} \int_{0}^{+\infty} \mathrm{d} X \int_{-\infty}^{+\infty} \mathrm{d} y \partial_{X} v_{i, 0} .
$$

Using $v_{i, 0}(+\infty, y, t)=v_{i, 0}^{g}(0, y, t)$, the definition of circulations $\Gamma_{i}, \Gamma_{i}^{g}$ in Eq. (3.33), and the notation $\left\langle\psi_{i}\right\rangle=\int_{0}^{+\infty} \mathrm{d} x \int_{-\infty}^{+\infty} \mathrm{d} y \psi_{i}$, integration of the r.h.s. in Eq. (4.4) yields

$$
\left\langle\psi_{i+1}+\psi_{i-1}-2 \psi_{i}\right\rangle=\operatorname{Bu} \delta z^{2}\left(\Gamma_{i}-\Gamma_{i}^{g}\right),
$$

The mass constraint in Eq. (3.35) follows from the equality $\Gamma_{i}=\Gamma_{i}^{g}$.

\section{REFERENCES}

BüHLER, O. 2000 On the vorticity transport due to dissipating or breaking waves in shallowwater flow. Journal of Fluid Mechanics 407, 235-263.

Deremble, B., Johnson, E. R. \& Dewar, W. K. 2017 A coupled model of interior balanced and boundary flow. Ocean Modelling 119, 1-12.

Dewar, W. K., Berloff, P. \& HogG, A. McC. 2011 Submesoscale generation by boundaries. Journal of Marine Research 69 (4-5), 501-522. 
Dewar, W. K. \& Hogg, A. McC. 2010 Topographic inviscid dissipation of balanced flow. Ocean Modelling 32 (1-2), 1-13.

HogG, A. McC., Dewar, W. K., Berloff, P. \& Ward, M. L. 2011 Kelvin wave hydraulic control induced by interactions between vortices and topography. Journal of Fluid Mechanics 687, 194-208.

MCWilliams, J. C. 1977 A note on a consistent quasigeostrophic model in a multiply connected domain. Dynamics of Atmospheres and Oceans 1 (5), 427-441.

Nguyen Van Yen, N., Waidmann, M., Klein, R., Farge, M. \& Schneider, K. 2018 Energy dissipation caused by boundary layer instability at vanishing viscosity. Journal of Fluid Mechanics 849, 676-717.

Peregrine, D. H. 1998 Surf zone currents. Theoretical and computational fluid dynamics 10 (14), 295-309.

Pratt, L. J. \& Whitehead, J. A. 2007 Rotating hydraulics: nonlinear topographic effects in the ocean and atmosphere, , vol. 36. Springer Science \& Business Media.

Rostami, M. \& Zeitlin, V. 2019 Eastward-moving convection-enhanced modons in shallow water in the equatorial tangent plane. Physics of Fluids 31 (2), 021701.

Roullet, G. \& Klein, P. 2010 Cyclone-anticyclone asymmetry in geophysical turbulence. Physical review letters 104 (21), 218501.

Roullet, G. \& McWilliams, J. C. 2014 2d turbulence with complicated boundaries. In $A G U$ Fall Meeting Abstracts.

Vallis, G. K. 2017 Atmospheric and Oceanic Fluid Dynamics. Cambridge University Press.

Zeituin, V. 2018 Geophysical fluid dynamics: understanding (almost) everything with rotating shallow water models. Oxford University Press. 